\title{
Soggettività e iterazione nel romanzo storico- metaforico di Vincenzo Consolo
}

\section{Gianni Turchetta}

\section{(2) OpenEdition}

1 Journals

Edizione digitale

URL: https://journals.openedition.org/cher/1159

DOI: $10.4000 /$ cher.1159

ISSN: 2803-5992

\section{Editore}

Presses universitaires de Strasbourg

\section{Edizione cartacea}

Data di pubblicazione: 6 novembre 2018

Paginazione: 29-54

ISBN: 979-10-344-0027-0

ISSN: 1968-035X

Notizia bibliografica digitale

Gianni Turchetta, «Soggettività e iterazione nel romanzo storico-metaforico di Vincenzo Consolo», reCHERches [Online], 21 | 2018, online dal 05 octobre 2021, consultato il 22 novembre 2021. URL: http://journals.openedition.org/cher/1159 ; DOI: https://doi.org/10.4000/cher.1159 


\section{Soggettività e iterazione nel romanzo storico-metaforico di Vincenzo Consolo}

GIANNI TURChETtA ${ }^{1}$

\section{Un (anti-)romanzo storico e un romanzo storico potenziale}

Nelle pagine che seguono mi propongo di indagare anzitutto alcuni aspetti fondamentali della poetica narrativa di Consolo: imprescindibili per capire la sua idea di romanzo storico-metaforico, e tuttavia non ancora studiati come dovrebbero. Essi rimandano a due fonti non solo storicamente comprovate, ma, di più, alla base di premesse di metodo decisive per la scrittura di Il sorriso dell'ignoto marinaio (Consolo 2015: 123-260) che di quelle premesse rappresenta la più compiuta e complessa realizzazione, e di altre sue opere. In seguito, proverò a mostrare alcuni aspetti strategici della densità e della ricchezza di stratificazioni testuali attraverso cui $\mathrm{Il}$ sorriso realizza una narrazione profondamente "poetica" anzitutto al livello delle macro-strutture, oltre che sul piano, più evidente e più spesso studiato, del linguaggio. Non è di per sé nuova l'idea di un romanzo dotato di una densità stilistica comparabile con quella della poesia, e certo Consolo la trovava già in uno scrittore di riferimento come Elio Vittorini: ma il modo in cui Consolo la realizza è originalissimo, e dà luogo a risultati di eccezionale intensità. Studierò poi, molto più sinteticamente, come anche Le pietre di Pantalica (Consolo 2015: 477-646), che a prima vista parrebbe un libro di racconti, mostri percettibilmente in filigrana proprio la struttura di un romanzo storico-metaforico affine per alcuni aspetti al Sorriso. I riscontri sono evidenti, e le carte d'archivio parlano molto chiaro: ma finora la bibliografia critica non ha in buona sostanza mai evidenziato come Le pietre di Pantalica sia nato da un progetto unitario, diventato in corso d'opera un libro diverso.

In prima approssimazione, Il sorriso dell'ignoto marinaio si presenta come un romanzo storico che affronta il dramma del Meridione a partire da un episodio

1 Gianni Turchetta, Università di Milano. 
sanguinoso del Risorgimento, la strage di Alcàra Li Fusi, criticando radicalmente gli esiti del Risorgimento, ma al tempo stesso evitando di cadere nel fatalismo anti-storico di Il Gattopardo: ragion per cui si è parlato spesso, motivatamente, del Sorriso come dell'anti-Gattopardo ${ }^{2}$. Consolo muove dall'esigenza di parlare di un fallimento storico, non destino eterno, ma frutto di un insieme di problemi politici, sociali ed economici, secondo una prospettiva evidentemente debitrice della lettura della storia italiana condotta da Antonio Gramsci nei Quaderni del carcere. Il sorriso parla però anche del presente, dei rischi di degenerazione della contestazione post ' 68 , e del problema della violenza politica, con riferimento alla tragica attualità del terrorismo. Anche da questo punto di vista, Consolo si rifà con ogni evidenza al grande modello del Manzoni, dei Promessi sposi ma anche della Storia della colonna infame, dove il passato allude al presente, secondo una dinamica sintetizzabile con una ben nota osservazione del narratore nel cap. VIII del capolavoro manzoniano: «Così va spesso il mondo... voglio dire, così andava nel secolo decimo settimo.»

D'altro canto, il Sorriso è però anche un romanzo programmaticamente anti-romanzesco, che contesta la forma stessa del romanzo, anzitutto sul piano strutturale, proprio mentre adopera il genere romanzo storico, che a sua volta già nasce come contestazione della storiografia. Consolo mette in discussione sia la storiografia, sia il romanzo storico, configurando il proprio romanzo come ridiscussione dei luoghi comuni storiografici e insieme negazione dell'impianto del romanzo storico, ma anche, più in generale, del romanzo tout court, in quanto narrazione filata, portatrice di illusione, nella sua adesione a una mistificata linearità degli eventi. Consolo vuole produrre un'impressione di realtà diminuendo la tradizionale mediazione narrativa, per proiettare il lettore nel flusso dello showing, cui corrisponde una massiccia erosione del telling (provocatoriamente confinato nelle Appendici documentarie e pseudodocumentarie). Ma al contempo egli mette radicalmente in discussione la macchina testuale che produce l'effetto di realtà, spezzandola, mescolandola con altri testi, e viceversa esibendo l'artificio. La mescolanza di vari generi testuali e di numerose voci narranti implica anche una vistosa relativizzazione delle voci. Dove la sceneggiatura narrativa delle molte voci si sovrappone all'esplicita messa in discussione della legittimità della voce narrante in genere e più specificamente delle voci supposte autorevoli: del protagonista, degli storiografi, degli estensori di documenti pubblici, di chi racconta la storia magari in buona fede, ma ricadendo inevitabilmente nella prospettiva dei vincitori, o quanto meno di chi ha il diritto di parola e il controllo degli strumenti di diffusione delle idee, cioè soprattutto della scrittura. Così infine, il Sorriso mette in discussione non solo il romanzo, e dunque la propria identità di genere, ma, in modo ancora più generale e più radicale, la parola degl'intellettuali, che sono tali perché hanno un certo livello economico-sociale, che fatalmente li avvicina al potere e li allontana dalla bruciante verità dei ceti non abbienti. Gl'intellettuali sono comunque vicini

2 Una definizione che era già stata attribuita a Il Consiglio d'Egitto di Leonardo Sciascia. 
al potere in quanto, direbbe Pierre Bourdieu, «frazione dominata della classe dominante»: è quindi necessario diffidare delle loro affermazioni. Lo sforzo quasi paradossale di Consolo consiste così nel provare a produrre una parola che possa sfuggire alla condizione privilegiata da cui nasce e alla mistificazione che ne consegue. Consolo sa bene che questo non è possibile, perché chi scrive è sempre in qualche modo già compromesso: ma proprio per questo deve parlare, e insieme mettere in discussione le proprie stesse parole, provando a mettere in scena il punto di vista di chi non può parlare. Da questo sforzo titanico deriva anche l'orientamento prevalente della sua ricerca linguistica, tesa a evitare la parola piatta, monocorde e monologica, dei mass media e della letteratura più compromessa con i gusti delle masse, per cercare, disperatamente, di dare voce alle voci di chi non ha strumenti per farsi sentire e sarebbe destinato a cadere nell'oblio.

Con Le pietre di Pantalica Consolo riprende la storia del Meridione italiano degli anni Quaranta, dallo sbarco americano in Sicilia nel 1943 alle lotte per l'occupazione delle terre nel dopoguerra, chiamando in causa poi direttamente anche il degrado degli anni Settanta-Ottanta. Le pietre di Pantalica mostra per certi aspetti una lingua più comunicativa, almeno in certe sue parti, e si presenta a prima vista come una raccolta di racconti, come già notato sopra. Ma in realtà, a ben guardare, ha una struttura composita: la prima parte, infatti, Teatro, che costituisce oltre la metà del libro, si rivela un corposo lacerto di romanzo storico, ben più che un abbozzo; il progetto di romanzo storico lascia tracce ancora nella seconda sezione, Persone; poi però sia gran parte della seconda sezione, sia la terza, Eventi, sono invece costituite davvero da racconti: più esattamente, da racconti di fiction, da racconti di impianto autobiografico e da testi fra saggistici e narrativi, vicini, sul piano del genere, al reportage giornalistico. L'analogia, anche sul piano delle dimensioni, fra questi testi e i primi, che corrispondono invece a episodi di una più ampia struttura narrativa, non deve però trarre in inganno. Infatti, l'impianto della prima parte delle Pietre di Pantalica mostra comunque, se guardata con maggiore attenzione, ancora vistosi elementi di continuità con il Sorriso, di cui riprende alcune modalità costruttive, specie al livello della macro-struttura narrativa: questa analogia è traccia vistosa di una continuità, che deve essere letta alla luce di scelte di poetica e procedimenti collocate alla radice del modo in cui Consolo concepisce il romanzo storicometaforico e più in generale la propria scrittura.

\section{Soggettivizzazione e «somiglianza»: dalle fonti al metodo compositivo}

Per capire un po' meglio il modo in cui Consolo realizza la straordinaria densità dei propri testi, è necessario mettere meglio a fuoco la peculiarità della sua sintassi narrativa. Sarei tentato di sostenere che il "segreto" del Sorriso è anzitutto sintattico: ma sarebbe affermazione troppo unilaterale. Certo però la sua particolarissima costruzione è un fattore decisivo, perché fa convivere meccanismi strutturali diversi, convergenti e al tempo stesso in competizione, 
che determinano un'intensità semantica straordinaria, che il testo comunica al lettore, obbligandolo a un lavoro di decodifica particolarmente intenso (la "difficoltà", che non è meramente lessicale, del testo consoliano): questa ricchezza semantica fa tutt'uno con la bellezza, con il valore estetico.

Non è mia intenzione trattare qui della molteplicità delle fonti del Sorriso: rimanderò per questo alla bella edizione critico-genetica di Nicolò Messina (2007) e agli apparati del Meridiano (Consolo 2015: XLI-LI, 1299-1319). Ma, come da premessa, mi soffermerò su due dei più importanti testi di riferimento per la poetica del Sorriso.

Anzitutto, il saggio di Hans Magnus Enzensberger Letteratura come storiografia, scritto appositamente per fare da introduzione al «Menabò»n. 9, sulla cui copertina spicca a mo' di titolo generale, in un numero dedicato a nove autori della narrativa sperimentale tedesca ${ }^{3}$, uscito poco prima dello scioglimento del Gruppo 47 (1947-1967). Questo numero del «Menabò», curato dallo stesso Enzensberger, progettato fin dal 1964 e uscito nel luglio 1966, è anche quello costruito durante la malattia di Elio Vittorini (mancato il 12 febbraio di quell'anno), nello sforzo vano di farglielo vedere finito. La lettura di Letteratura come storiografia rappresenta sicuramente un momento decisivo per la progettazione del Sorriso, che Consolo sta avviando, e con cui mostra vistosissime consonanze molto specifiche e profonde. Riprendendo Herder, secondo il quale «la letteratura è la vera storiografia dell'umanità» (p. 13), Enzensberger afferma senza mezzi termini che «Il solo coerente sistema di segni, da cui può essere colta la storia come realtà materiale, sembra essere la letteratura» (p. 12). Quest'affermazione è nota; meno nota però è l'insistenza con cui Enzensberger prepara l'affermazione, opponendo la pretesa obiettività di una storiografia in cui «La storia viene esibita senza il suo soggetto» (p. 8) ai modi rappresentativi della letteratura, dove «Tutto quello che avviene ha un destinatario per il quale avviene. È avvenuto così ma per chi?» (p. 9). L'opposizione viene messa a fuoco contrapponendo un passo di Berlin Alexanderplatz, caratterizzato dalla prospettiva totalmente soggettivizzata di Franz Biberkopf, a un passo di Storia della Germania del XIX e del XX secolo di Golo Mann. Commenta Enzensberger:

Döblin si tiene fermo alla sua paternità di autore proprio là dove egli la delega e il suo stile personale diviene evidente in massimo grado dove egli cita. Non si evita la lingua burocratizzata, che viene ripresa in molteplici variazioni, per lo più senza commento. In stridente contrasto le vengono messe accanto altre lingue. [...] In questa Babele di lingue soggettive e obiettive, l'una critica e mette a nudo l'altra. (Enzensberger 1966: 10)

3 Il numero comprende dieci testi di altri nove scrittori: Karl Mundstock, Peter Hacks, Hans Günter Michelsen, Alexander Kluge, Arno Schmidt, Martin Walser, Jürgen Becker, Peter Weiss, Uwe Johnson. Per non appesantire l'apparato di note, anche nelle citazioni del saggio di Enzensberger mi limiterò a indicare fra parentesi il numero di pagina. 
Segue poco dopo l'affermazione già citata, che Consolo ricorderà per sempre, sulla letteratura come «storiografia», e l'ulteriore sviluppo dell'argomentazione, secondo la quale le opere di Bertolt Brecht sono «una parte di storiografia tedesca», proprio perché impongono il rovesciamento della consueta prospettiva della storiografia: si pensi, per esempio, a un testo poetico celeberrimo come Domande di un lettore operaio. I testi di Brecht, continua Enzensberger, ci dicono «Non come sono andate le cose, ma come sono andate per i muratori» (p. 13), che normalmente non hanno diritto di parola. La letteratura non è stata di per sé con i potenti, ma è stata sempre «limitata da interessi nazionali e di classe, contrassegnata e circoscritta già dal fatto che la sua esistenza presuppone l'agio, quindi la ricchezza, quindi lo sfruttamento» (p. 14). Siamo, molto visibilmente, proprio sulla strada che sarà poi percorsa dal Sorriso, e in particolare, con queste ultime affermazioni, già sulle soglie delle analoghe, esplicite considerazioni del barone Mandralisca nel cap. VI. Inoltre, secondo Enzensberger, «d'ora in poi la letteratura è possibile solo in quanto crisi della letteratura stessa» (p. 21). Le consonanze con Consolo sono davvero molto evidenti, su vari piani. Vale la pena di riprendere in dettaglio le proposte di Enzensberger, perché si ritrovano punto per punto nelle scelte formali (strutturali prima che stilistiche) di Consolo:

- La soggettivizzazione programmatica della rappresentazione;

- La coscienza della relatività degli stessi documenti storici, a loro volta fatalmente toccati dalla soggettivizzazione (cfr. Enzensberger 1966: 22);

- La citazione sistematica delle lingue altrui, fatte cozzare fra loro, ma proprio per dare luogo alle marcatissime peculiarità della lingua autoriale;

- La pluralità e correlativamente la forza relativizzante delle diverse lingue, che sono sempre anche prospettive (una linea poetica e operativa, vale la pena di notarlo, perfettamente convergente con quanto Bachtin aveva già scritto negli anni Trenta, ma in Italia avremmo letto solo dopo il 1979);

- La messa in scena delle prospettive di chi non può parlare;

- L'affermazione della forza di verità della letteratura, che fa tutt'uno con la sua "dannazione", con la sua compromissione col potere, e dunque con la necessità paradossale di fare una letteratura che neghi se stessa.

Il secondo riferimento è a prima vista scontato, ma merita di essere più specificamente interpretato in relazione alla pratica di scrittura che suggerisce. Si tratta del saggio di Leonardo Sciascia L'ordine delle somiglianze, di cui un passo costituisce la seconda epigrafe del Sorriso:

Il giuoco delle somiglianze è in Sicilia uno scandaglio delicato e sensibilissimo, uno strumento di conoscenza [...] I ritratti di Antonello "somigliano"; sonol'idea stessa, l'arché, della somiglianza [...] A chi somiglia l'ignoto del Museo Mandralisca? Leonardo Sciascia, L'ordine delle somiglianze (Consolo 2015: 125)

Il saggio nasce come presentazione a L'opera completa di Antonello da Messina (Sciascia 1967). L'avvio della parte che ci interessa dell'argomentazione di Sciascia deriva in realtà da affermazioni di Antonio Castelli, scrittore cefalutano poco conosciuto ma di grande valore, autore di testi narrativi di rilievo come Gli ombelichi tenui (1962) e Entromondo (1967), cui lo stesso 
Sciascia e Consolo avrebbero cercato di far vincere il Premio Brancati nel $1968^{4}$. Rileggiamo per esteso il passo in questione di Sciascia:

ad aprire (e forse, effettualmente, a chiudere) il nostro breve discorso su Antonello, ci soccorre questa acuta notazione di Antonio Castelli, il quale, per essere nato a Cefalù, non è improbabile sentisse nello scriverla, vagamente e sottilmente, il suggerimento di quel prodigioso ritratto di Antonello che si trova nel cefalutano Museo Mandralisca: "Nella comunità alla quale apparteniamo, nel paese dove nasciamo, risiede la nostra nozione del colore; e la nostra misura d'uomo è regolata su un ordine bioetnico delle somiglianze. Sono l'assoluto fisiognomico e l'assoluto cromatico, calati nel crogiuolo della terra natia, a modulare il nostro consistere".

"L'ordine bioetnico delle somiglianze", da cui scatta "l'assoluto fisiognomico": sono espressioni che immediatamente ci collegano ai personaggi d'Antonello. Anche ai santi. Anche alle Madonne.

Il giuoco delle somiglianze è in Sicilia uno scandaglio delicato e sensibilissimo, uno strumento di conoscenza. A chi somiglia il bambino appena nato? A chi il socio, il vicino di casa, il compagno di viaggio? A chi la Madonna che è sull'altare, il Pantocrator di Monreale, il mostro di villa Palagonia? Non c’è ordine senza le somiglianze, non c'è conoscenza, non c'è giudizio. I ritratti di Antonello 'somigliano'; sono l'idea stessa, l'archè, della somiglianza. A ciascuno si possono adattare tutte le definizioni che sono state date dei siciliani, da Cicerone a Tomasi di Lampedusa: sono chiusi sospettosi sofisti; amano contraddirsi e contraddire, complicare le cose con l'astuzia e risolverle con secco intelletto; sono sensuali avidi violenti, tesi al possesso della donna e della roba, ma in ogni loro pensiero è annidata accettata vagheggiata la morte.

A chi somiglia l'ignoto del Museo Mandralisca? Al mafioso della campagna e a quello dei quartieri alti, al deputato che siede sui banchi della destra e a quello che siede sui banchi della sinistra, al contadino e al principe del foro; somiglia a chi scrive questa nota (ci è stato detto); e certamente somiglia ad Antonello. E provatevi a stabilire la condizione sociale e la particolare umanità del personaggio. Impossibile. È un nobile o un plebeo? Un notare o un contadino? Un uomo onesto o un gaglioffo? Un pittore un poeta un sicario? 'Somiglia', ecco tutto. (Sciascia 1998: 35-36)

Al di là della localizzazione siciliana, è necessario tenere in particolare conto l'affermazione apodittica di Sciascia per cui, in generale, "Non c'è ordine senza le somiglianze, non c'è conoscenza, non c'è giudizio.» Prima di procedere oltre, è necessario fare il punto sulle date. Il saggio in questione di Enzensberger e quello di Sciascia, pubblicati a pochissima distanza, vengono sicuramente letti da Consolo subito a ridosso della loro uscita. Cronologicamente, si collocano già a una certa distanza dall'esordio di La ferita dell'aprile, e, soprattutto in prossimità della scrittura del primo capitolo del Sorriso, di cui una prima versione sarà spedita a «Paragone» nel 1968, senza ottenere risposta, e qualche mese dopo

4 Si tratta di una vicenda molto rilevante della biografia di Consolo, da cui scaturì una polemica con Corrado Stajano che sarebbe poi diventata l'avvio della loro profonda amicizia. Cfr. Turchetta 2015b: CXVI. 
anche a «Nuovi Argomenti», che l'avrebbe pubblicata nel numero estivo del $1969^{5}$. Non a caso, i testimoni antiquiores dell'elaborazione del testo, cioè i tre quaderni manoscritti denominati MS1, MS2 e MS2bis (secondo la recensio dell'edizione di Nicolò Messina, ripresa nel Meridiano), sono sicuramente anteriori al testo preparato per «Nuovi Argomenti», e con ogni probabilità anche a quello spedito a "Paragone». Altri indizi ci riportano senza possibilità di dubbio proprio agli stessi anni dei due testi di Enzensberger e Sciascia, confermando indirettamente che la fase decisiva per la concezione del Sorriso si colloca proprio fra il 1966 e il 1968. Uno fra tutti: sul f. 17 di MS2, dopo appunti che paiono un programma di scrittura del cap. I del Sorriso, sull'ultima riga, poi cassata, leggiamo: «Foucault. Descrizione di un quadro di Velázquez»; evidentemente Consolo sta leggendo il cap. I della prima parte di Le parole e le $\cos e^{6}$, che si mostra a sua volta rilevante per la genesi del passo che troviamo subito dopo nel manoscritto: una descrizione del ritratto di Antonello, riscritta con limitate correzioni.

L'epigrafe di un libro, si sa, non è una citazione come tante. In particolare, in questo caso la citazione del passo di Sciascia non può essere letta solo in riferimento alla circostanza narrativa, a cui pure va ricollegata, e che potrebbe persino avere ispirato; si tratta, per lo più, di una circostanza fondamentale anche a livello d'intreccio: mi riferisco ovviamente alla somiglianza prodigiosa dell'Ignoto del ritratto con Giovanni Interdonato, che Mandralisca incontra nel capitolo I per caso sulla nave con cui porta il quadro a Lipari, e che in seguito andrà a trovare lo stesso Mandralisca per chiedergli un più fattivo impegno a favore dei moti risorgimentali. Questa somiglianza prodigiosa, se limitata a un mero meccanismo diegetico, rischia di apparire come un artificio romanzesco nel senso deteriore del termine. A rigore, non possiamo escludere del tutto questa possibilità, che del resto s'intona perfettamente con l'impiego demistificatorio, quando non parodico, dei meccanismi romanzeschi da parte di Consolo. Tuttavia, proprio la collocazione strategica del riferimento all'“ordine delle somiglianze" in epigrafe, impone interrogativi di fondo relativi non solo al misterioso uomo del ritratto, e neanche solo alla questione esplicitamente enunciata, quella cioè della "somiglianza" in Sicilia. Più in profondità, Consolo allude, senza possibilità di dubbio, al rapporto fra rappresentazione artistica e realtà: con ogni evidenza, infatti, l'interrogativo su quello specifico quadro si amplia a interrogativi più generali, sottolineando inoltre una prima fondamentale relazione di analogia/differenza, somiglianza/eterogeneità fra la pittura e la letteratura. Questa relazione problematica si riproporrà molte volte nel libro, specie nei passi

5 Per la vicenda della pubblicazione del Sorriso, si vedano Turchetta 2015b: CXVII, CXXII-CXXIII e Turchetta 2015c: 1299-1303.

6 Foucault 1978: 324. Quest'ultima edizione è quella rimasta presente nella biblioteca di Consolo, con sottolineature a matita; ma la citazione di MS2, certo anteriore alla pubblicazione del primo capitolo su Nuovi Argomenti, non lascia dubbi: Consolo sta leggendo, se non l'originale, almeno la prima edizione italiana, del 1967. 
di ekphrasis, che nelle edizioni in volume singolo del romanzo non smettono di rimandare alla copertina, dove è di norma fisicamente riprodotto il quadro di Antonello. In tutta la sua opera, del resto, Consolo pone il problema dei rapporti fra arte e realtà. In questo modo egli chiama in causa, più ampiamente, la forza generalizzante della rappresentazione, quella peculiare miscela di concreto e astratto che è alla base della forza simbolica dell'arte. Anche questo tuttavia è ancora poco, o comunque non abbastanza, è necessario andare ancora più in là. Una folla di dati testuali ci segnala infatti che L'ordine delle somiglianze, o, detto in altri termini, l'accostamento di figure, temi e in genere elementi testuali simili costituisce per Consolo un principio compositivo fondamentale, che l'epigrafe sciasciana annuncia a mo' di dichiarazione di poetica. Come cercherò di mostrare qui di seguito, tutta la sintassi narrativa del Sorriso si regge sistematicamente su un corposo, ben visibile "ordine delle somiglianze": nel senso che procede stabilendo una fittissima serie di corrispondenze e analogie, di rimandi incrociati e sovrapposizioni, che di fatto fanno funzionare il testo secondo due principi strutturali opposti. Da un lato, infatti, e, verrebbe da dire, nonostante tutto, il testo si sviluppa secondo una logica diegetica, di progressione narrativa, sull'asse sintagmatico. Da un altro lato il costituirsi di continue corrispondenze istituisce una logica invece di carattere prevalentemente poetico, in cui la sintassi accosta vistosamente elementi costruiti in modo simile, con un principio affine a quello dell'accostamento di unità metrico-ritmiche in poesia. La contestazione della linearità narrativa si realizza anche e proprio nella marcata poetizzazione strutturale, che accentua in modo sostanziale lo stesso pervasivo metaforismo: cioè la tensione generalizzante, mitologizzante, che si intreccia ad ogni passo con la fittissima trama documentaria, che spinge invece nella direzione contraria, dell'individuazione e della storicizzazione.

In questo modo il discorso conserva un ben percepibile asse narrativo, che dobbiamo decodificare per seguire la storia, ma al tempo stesso, attraverso il gioco delle somiglianze, crea una dinamica che nega l'andamento lineare, una dinamica in cui la successione sintagmatica giustappone elementi simili o resi simili, creando nuove corrispondenze paradigmatiche, che assumono contemporaneamente valenze lato sensu ritmiche, di ripetizione di unità testuali analoghe, imponendo a ogni unità testuale elementi di senso che appartengono alle altre unità analoghe e dando così luogo a nuove stratificazioni di significato. Questa dinamica viene appunto determinata dalla sistematica attivazione, da parte di Consolo, di "somiglianze", via via costituite dalla progressione del testo, che accosta e rende analoghi elementi in prima approssimazione non equivalenti, eterogenei, producendo nuovi strati di senso ${ }^{7}$. Si viene a creare una correlativa regola strutturale, costituita proprio dall'accostamento di elementi diversi, cui viene assegnata la marca della somiglianza. La somiglianza impera, ma si marca a partire dalla differenza, dall'eterogeneo. Il risultato strutturale è

7 Sul piano teorico, faccio qui riferimento a Lotman 1972-1980, in particolare al cap. V, I principi costruttivi del testo, p. 101-119. 
che, in maniera analoga a quanto avviene in poesia, «l'equazione serve a costruire la successione», o, detto in altro modo, «il principio d'equivalenza» si proietta "dall'asse della selezione all'asse della combinazione», così che «l'equivalenza è promossa al grado di elemento costitutivo della sequenza» (Jakobson 1981: 192. Corsivi nel testo). Sono, lo so, affermazioni molto note agli studiosi, che sono state anche giustamente criticate in profondità, quanto meno nella loro pretesa normativa. Tuttavia, esse continuano a descrivere molto bene il funzionamento del linguaggio poetico, e non a caso un testo capitale come quello di Lotman vi fa riferimento in modo sistematico. Nel nostro caso tuttavia, colpisce, ma non sorprende, che esse riescano a descrivere perfettamente non un testo poetico, ma un romanzo, il cui peculiare funzionamento strutturale è vistosamente analogo a quello di una poesia.

La folla dei rimandi interni del Sorriso, su cui fra poco mi soffermerò in dettaglio, crea un gioco fittissimo di corrispondenze, di "somiglianze", che si proiettano dall'asse paradigmatico all'asse sintagmatico. La compresenza di meccanismi narrativi e poetici, già al livello della macro-struttura, produce inoltre un altro effetto. Mi appoggerò ancora a Lotman: in ogni testo convivono un aspetto "fabulistico", mediante il quale un testo rappresenta specifici aspetti della realtà, e un aspetto "mitologico", "con cui il testo simula un intero universo” (Lotman 1972-1980: 255). La struttura del Sorriso riesce a intensificare insieme l'uno e l'altro aspetto: la storia e la metafora, appunto. Questa dinamica fondamentale genera un aumento esponenziale di complessità strutturale, un aumento che dà luogo a una eccezionale densità semantica del testo e a una correlativa "difficoltà". Infatti,

Proprio perché quanto più complessa è l'organizzazione del testo e di ogni suo livello, tanto più imprevisti sono i punti di intersezione delle sottostrutture particolari; quanto maggiore è il numero delle strutture nelle quali è inserito un dato elemento, tanto più «casuale» esso ci sembrerà, si ottiene un noto paradosso peculiare del testo artistico: un aumento di strutturalità porterà a una diminuzione di prevedibilità. (Lotman 1972-1980: 323)

\section{Le strutture della somiglianza: modularità e ritmicità}

Finora mi sono limitato ad affermazioni di carattere generale, che potrebbero anche parere un po' astratte. I dati testuali le supportano tuttavia in modo assai robusto, innegabile, confermando, a molti livelli, un metodo costruttivo dove la sintassi narrativa è massicciamente tramata da strutture analoghe, che danno luogo a un regime di "somiglianze" capillare e pressoché generalizzato. Anzitutto, anche a uno sguardo corsivo, praticamente ad apertura di libro, risalta la sistematica, calcolata frammentazione della struttura del Sorriso. Sarebbe più corretto però parlare di segmentazione, anzi, più radicalmente, di una modularità, cioè della costruzione del testo mediante la collocazione in serie di unità narrative analoghe, in prima approssimazione addirittura potenzialmente interscambiabili. La progettazione modulare del Sorriso trova 
robusti e inequivoci riscontri nell'iter dell'elaborazione testuale. Spiccano le carte raccolte in una cartelletta denominata DS 1, comprendente dodici fascicoli, numerati da DS1.0 a DS1.11 (Messina 2007, Turchetta 2015c: 1315-1317). Un ruolo strategico spetta al fascicolo Ds1.1, "Carte per gioco», che segna il passaggio definitivo dalle prime versioni dei capitoli I e II, che appaiono quasi come racconti paralleli, a un più ampio progetto romanzesco, per quanto ancora in uno stato di elaborazione relativamente embrionale. È un testo chiaramente posteriore alla morte di Lucio Piccolo (26 maggio 1969) e certo anteriore alla prima edizione, in un prezioso volume d'arte, dei capitoli I e II (Consolo 1975). Consta di 13 ff., e ipotizza una narrazione tripartita, in tre «tempi»: "Primo tempo» (ff. 1-4): dove troviamo parti del cap. I, l'Antefatto e l'Appendice I; «Secondo tempo» (ff. 4-10), con documenti storici, poi in parte riportati nel cap. IX, nelle Appendici I, II, III; «Terzo tempo» (ff. 11-13), che avrebbe dovuto essere dedicato a Piccolo e rappresentare un'area privilegiatamente poetica del testo, e che riprende l'articolo Il barone magico del $1967^{8}$. A quest'altezza cronologica, la tripartizione assume palesemente anche la forma di una distribuzione per generi:

CARTE PER GIOCO

(Racconti e cose da raccontare fin dal tempo di Garibaldi)

BLASONE DI CEFALU

Primo tempo - Narrativo. (a mo' di raeconto).

[...]

Secondo tempo - Storico.

I quaranta giorni di anarchia della città di Alcara Li Fusi sopra i Nebrodi.

$[\ldots]$

Terzo Tempo - Magico (o poetico)

In ricordo del barone

Lucio Piccolo di Calanovella

Autore dei Canti Barocchi ${ }^{9}$

In questo stadio di elaborazione, Consolo pare pensare a una sequenza in cui compare prima la fiction, poi la documentazione storiografica, infine la poesia. Il titolo ipotizzato sembra rimandare peraltro al citato Consiglio d'Egitto di Leonardo Sciascia. Anche se abbandonato nella sua versione più radicale, che prevedeva addirittura una relativa intercambiabilità dei capitoli, questo progetto modulare lascia comunque tracce vistose nell'impianto definitivo del romanzo. L'asse diegetico del romanzo viene bloccato, ma resiste la relativa equivalenza delle parti, più marcata per le parti diegetiche, ma percepibile in parte anche nelle Appendici. Nella versione finale Il sorriso viene suddiviso in sedici parti, di cui nove capitoli diegetici e sette testi di appendice documentaria. L'articolazione molto segmentata viene a configurare una sorta di elementare scansione ritmica,

8 Consolo 1969, poi ripreso quasi integralmente in Il barone magico, I, in Consolo 2015: 601-605.

9 Vincenzo Consolo, DS 1.1, in Archivio Consolo; riportato anche in Turchetta 2015c: 1315. 
dove i capitoli costituiscono qualcosa di molto simile alle lasse di una struttura poematica, cui alludono (e che non a caso sarà del tutto conclamata nelle opere consoliane degli anni Novanta, su cui tornerò brevemente alla fine). Da questo punto di vista, e non solo, il cap. IX ripete e ribadisce la struttura complessiva, di cui costituisce una sorta di mise en abyme. La scansione modulare è infatti chiaramente reiterata all'interno del cap. IX, integralmente composto dai graffiti dei carcerati, che hanno, se presi singolarmente, l'aspetto indiscutibile di poesie, dove gli a capo, in mancanza di motivazioni "materiali", somigliano irresistibilmente a degli a capo di fine verso; se presi insieme, i dodici graffiti appaiono anche come le lasse di un poema unitario, che, com'è noto, allude alle dodici stazioni della Via crucis, peraltro già evocate nel cap. VII, a ulteriore conferma della disseminazione onnipervasiva dei meccanismi iterativi.

Le scansioni modulari vengono ulteriormente ribadite dall'evidenza di ulteriori partizioni simmetriche, che raggruppano i moduli (capitoli e/o Appendici) per gruppi di due o tre. Tornerò più avanti sull'evidente tendenza a collocare anche i personaggi in coppie simmetriche. Sul piano macro-strutturale, le simmetrie sono particolarmente vistose nel "pacchetto" delle Appendici (saggistiche e documentarie, comunque composte da testi di non fiction). Le sette appendici appaiono così di fatto raggruppate in tre gruppi: due per il cap. I, due per il cap. II, tre per il cap. IX. Ciò significa inoltre che le Appendici sono tutte concentrate agli estremi del testo, e dunque sul suo marcatissimo confine: un aspetto che meriterebbe a sua volta una trattazione analitica, che mi riservo di sviluppare in altra sede. Di fatto, abbiamo quindi ancora una struttura con dodici elementi: nove capitoli diegetici + tre blocchi di Appendici (né c’è bisogno di essere maniaci di numeri per notare che sia il 12 sia il 9 sono multipli di 3). Una struttura, e una numerologia, vistosamente analoga a quella reiterata dal cap. IX e ultimo. Il numero 12 rimanda in modo evidente a un'antica e diffusa simbologia religiosa (fondata sulla moltiplicazione del 2 e del 3, cioè, più esattamente, di $2^{2} \times 3$ ), cui Consolo allude spesso, o comunque adopera, consapevolmente o inconsapevolmente: se nel Sorriso ci sono $9(+3)$ capitoli e 12 scritte dei carcerati (che sono anche 12 martiri, e 12 testimoni, come gli apostoli oltre che come le stazioni della Via crucis), in dodici capitoli sono articolati anche La ferita dell'aprile e Nottetempo, casa per casa; ma a sua volta Lo Spasimo di Palermo è composto da un Proemio e da undici capitoli, quindi da dodici capitoli di fatto. Meno significativa, ma ancora più insistente, è poi la disposizione per schemi ternari, già ricorrente nel Sorriso (dove si ripresenta ripetutamente in varie sedi, come i blocchi di Appendici, e in vari fasi dell'elaborazione testuale, come in parte abbiamo visto), e ripresa al livello macro-strutturale, in modo particolarmente vistoso, sia in Retablo sia in Le pietre di Pantalica.

In sintesi: la struttura modulare costruisce il testo per via di segmenti simili, secondo una regola approssimata di "somiglianza" già di per sé iterativa, portatrice di una ritmicità ben percepibile, anche se sotto traccia. Su questa trama complessiva la "numerologia" consoliana dà luogo ad ulteriori simmetrie, 
associando i segmenti in modo tale da disegnare altre "somiglianze", per così dire, di secondo grado.

\section{La soggettivizzazione dei resoconti}

Alla struttura modulare, incipientemente ritmica, si accompagna la pratica sistematica della soggettivizzazione, che intreccia il principio della somiglianza con la pratica della letteratura come "vera storiografia", cioè come storiografia che non oblitera le soggettività di chi vive i fatti narrati, in linea con le indicazioni di Enzensberger. Come abbiamo visto, la soggettivizzazione del testo, o meglio dei suoi numerosi componenti, si reitera nell'adozione di una pluralità non solo di voci, ma anche di modi narrativi e di generi, con la conseguente radicale, sistematica relativizzazione di ogni discorso. Il risultato, con ogni evidenza, va nella direzione di una vistosa polifonia, che implica, come già notato da Cesare Segre sulla scia di Bachtin, non solo pluri-linguismo, ma anche plurivocità, cioè, strategicamente, pluralità di prospettive e di ideologie (Segre 1995: I-XIX).

In prima approssimazione, come visto, Il sorriso consta di nove capitoli diegetici e sette Appendici, raccolte in tre blocchi. Se guardiamo meglio, però, i capitoli propriamente diegetici sono solo i primi cinque. Il cap. VII è infatti diegetico, ma su una linea lato sensu saggistica, perché presentato con la forma documentaria della Memoria, che allude vistosamente ai trattati scientifici redatti dal Mandralisca, e citati, ma come veri documenti, nelle due Appendici del cap. I. I capp. VI e VIII complicano e confondono ulteriormente le scelte di genere, dal momento che si configurano rispettivamente come una lettera e come una sorta di introduzione saggistica: anche se sul piano del contenuto sono testi pienamente di fiction. In particolare, lo strategico cap. VI appare, a livello di genere, analogo all'Appendice prima del cap. I, che pure è una lettera, ma vera, di Mandralisca; laddove il cap. VII assomiglia a una versione molto allargata, oltreché a una apostasia, dell'Appendice seconda del cap. I. Quanto al IX, ne abbiamo già commentato la peculiarità, per non dire la "stranezza" strutturale. Di fatto, a partire dal cap. VI, la diegesi vera e propria lascia il campo piuttosto a pseudo-documenti, che vanno a sovrapporsi con i documenti veri e propri, creando ulteriore confusione, o, detto un po' meglio, a livello macro-strutturale, nuovi importanti fenomeni di somiglianza/eterogeneità, che rimescolano le carte anche e proprio a livello di genere.

Contemporaneamente, la mescolanza narratore esterno/focalizzazione interna lascia il passo all'attribuzione piena della funzione di voce narrante al Mandralisca, che diventa narratore e prende stabilmente la parola proprio mentre mette in atto un'esplicita procedura di auto-delegittimazione. L'idea di una letteratura che può essere se stessa, e adempiere ai propri doveri di verità storica, prende corpo così, visibilmente, nel paradosso di un narratore che si rende autorevole proprio dichiarando senza remore la propria fatale inattendibilità. D'altro canto, anche se Mandralisca ne prende nell'ultima parte in mano il filo, la trama narrativa del Sorriso resta composta da una molteplicità 
particolarmente accentuata di voci, che registra quasi una folla di narratori, o, più esattamente, di responsabili dell'enunciazione (spesso non propriamente narrativa), i quali, in linea con il paradigma manzoniano della mescolanza di "storia" e "invenzione", sono sia di fiction sia realmente esistiti. In un rapido elenco, le voci principali sono, in ordine di apparizione, e tenendo conto sia dei capitoli diegetici sia delle Appendici: il narratore esterno, con un corredo ricchissimo di focalizzazioni interne, che delegano la visione, e spesso anche la voce, ad altri personaggi, cominciando, massicciamente, dal Mandralisca "inventato"; il Mandralisca "vero", dei documenti; Francesco Guardione; Giuseppe Cesare Abba; il Mandralisca di fiction; i nove autori dei graffiti; Luigi Scandurra; Giuseppe Natoli Calcagno, ufficiale dello stato civile; Antonio Mordini. Ancora più complessa è la fenomenologia di sovrapposizioni, cioè ancora di somiglianze e diversificazioni, tra i soggetti dell'enunciazione e i generi testuali. Ma ben più ampio sarebbe l'elenco se vi comprendessimo i numerosi narratori e / o responsabili delle narrazioni/ enunciazioni di secondo grado, cui la parola viene affidata nel testo in maniera mediata, attraverso, fra le altre, a procedimenti come il dialogo e la citazione prolungata. Anche questi danno luogo a una reiterazione dei fenomeni di sovrapposizione fiction vs. non fiction, complicando ulteriormente il quadro dei rapporti enunciativi, e in specie le dinamiche fra discorso primo e discorso riportato e/ o citato. Per dare un'idea approssimata ma certo utile a farsi un'idea di questa complessità, propongo un rapido censimento solo delle voci principali individuabili nettamente (lasciando da parte i personaggi che prendono la parola nei dialoghi), all'interno del resoconto prendono "di fatto" la parola, con modalità diverse: Saverio Landolina; Giovanni Interdonato, che racconta di nuovo le circostanze dell'Antefatto a Mandralisca; Francesco Carrera, autore del Pantheon Siculum sive Sanctorum Siculorum Eulogia, verosimilmente citato attraverso Gaetano Morelli, a sua volta autore di San Nicola Politi patrono di Alcara ${ }^{10}$; il narratore dei Promessi sposi, che eviteremo di confondere con Alessandro Manzoni; Mario Pagano e Carlo Pisacane; Filippo Buonanni; Plauto, e persino Archimede.

Ancora più capillarmente, tutta la fenomenologia delle citazioni, dell'intertestualità, lungi dall'essere un mero fenomeno di rimando intertestuale e di erudizione, andrebbe inquadrata anche e proprio in questa prospettiva, di rapporti fra soggetti enunciativi. In questo quadro, particolarmente rilevante è la sovrapposizione fra testi citati, e colti, e parole/ pensieri di personaggi incolti o semicolti. In particolare, in modo assai vistoso, nel cap. III, con frate Nunzio, ma anche nel cap. V, con la focalizzazione su Giuseppe Sirna Papa, i cui pensieri si mescolano a quelli dell'Innominato nella notte drammatica della conversione ${ }^{11}$. Nella costruzione di Consolo gioca inoltre un ruolo molto importante, ancora più radicalmente, la focalizzazione interna come mimesi dell'esperienza nel suo farsi. Oltre a sovrapporre più visioni del mondo incompatibili, con una radicalità

10 Come ha mostrato brillantemente Messina 2007.

11 Alessandro Manzoni, I promessi sposi, cap. XXI. 
intransigente che ha pochi termini di comparazione, almeno in Italia, Consolo ci pone di fronte a un intreccio vertiginoso fra resoconto narrativo e voci dei personaggi, che si complica vistosamente con l'intreccio fra le voci pronunciate e, per così dire, le voci prima della voce, i discorsi prima dei discorsi, i pensieri ma anche le percezioni non formate. Il lettore si trova così di fronte a parole che scendono sistematicamente al tempo e ai modi che ne precedono la formulazione. L'esigenza di verità della parola letteraria si fa in questi casi esigenza di rivivere tutto dall'interno, di entrare nel cuore dell'esperienza dei personaggi, di arretrare fin là dove l'esperienza si sta formando, nel luogo dinamico dove sono le cose prima di essere nominate, le percezioni, i sentimenti prima di essere coscienti, $\mathrm{i}$ gesti non ancora realizzati ${ }^{12}$.

Alla moltiplicazione delle prospettive fa riscontro una narrazione che in molte occasioni narra più volte lo stesso fatto, configurando, per riprendere la terminologia di Genette, un racconto ripetitivo, dove un evento viene narrato $n$ volte. Ciò significa però anche esibire la parzialità, la prospetticità inevitabile di ogni singola narrazione di uno stesso fatto. Si comincia con lo sfregio inferto da Catena Carnevale al quadro di Antonello, narrato nell'antefatto e nel cap. II. Poi c'è la presenza di Interdonato sulla nave con cui Mandralisca porta il ritratto da Lipari. Ancora, l'arrivo di Interdonato è narrato nel cap. II e ripreso nel cap. VI. Anche l'arrivo dei garibaldini è raccontato a più riprese, così come la stessa morte violenta della "vergine" per mano di frate Nunzio, al cap. III e al cap. V. Particolarmente interessante è proprio il caso esemplare, sul piano narrativo, sul piano storiografico e anche su quello gnoseologico, della rivolta di Alcàra Li Fusi, che, anche se in prima approssimazione rimane fuori scena, viene di fatto narrata di scorcio più volte: a partire dalla preterizione del cap. VII, ma conquistando il primo piano solo nei graffiti/poesie dei carcerati del castello gallego, apoteosi del soggettivismo tematico ed espressivo, nel capitolo finale.

Molto importante per la scansione macro-strutturale delle somiglianze è poi la vistosa scansione per blocchi di quattro anni dei primi capitoli: il cap. I infatti si svolge nel 1852, il cap. II nel 1856, il cap. III nel 1860. Siamo evidentemente alle prese con una narrazione che procede per scene, sottoponendo a ellissi gran parte degli eventi, almeno per quanto riguarda la diegesi vera e propria. Sono infatti le Appendici a fornire il contesto, consentendo al lettore la ricostruzione degli eventi. Ma non meno evidente è il costituirsi di un'ulteriore chiara simmetria, che è già quasi un ritmo. Notiamo già, anticipando, che la scansione per quattro anni si ripete anche nella prima sezione di Le pietre di Pantalica, che nel primo blocco di testi si dedica a eventi del 1943, passando nel secondo blocco a eventi del 1947. Non è peraltro certo un mero fatto formale, anche se pare quasi un'ossessione, il ripetersi, da un libro all'altro, della rappresentazione dello sbarco in Sicilia: prima dei garibaldini, poi degli americani.

12 A questo proposito, mi permetto di rimandare a Turchetta 2016: 535-565. 


\section{Le corrispondenze fra personaggi}

La costruzione per somiglianze si manifesta a molti livelli, e appare anzi poco meno che onnipervasiva. Anche l'invenzione centrale della fabula, come già accennato, si fonda su un caso conclamato e quanto mai "romanzesco" di somiglianza: quella, già ricordata e fondamentale, per cui l'Ignoto di Antonello "somiglia" incredibilmente a Giovanni Interdonato; e dunque, si badi bene, una rappresentazione artistica "somiglia" a un individuo "reale" (che in un certo senso, ancora più incredibilmente, quasi pare derivarne, dal momento che compare poco meno di quattrocento anni dopo), che però è a sua volta personaggio di romanzo, ma realmente esistito e trattato anche dalla storiografia, ora mostrato nel contesto di un romanzo che contesta sia il romanzo sia la storiografia, rimettendo in discussione radicalmente lo statuto dell'arte ma anche quello della realtà. Un gioco di specchi, come si vede, vertiginoso: ma tutt'altro che formalistico. Questa somiglianza si colloca però, di nuovo, in una fitta trama di ulteriori "somiglianze", analogie e simmetrie fra i personaggi del romanzo: un dato particolarmente suggestivo del Sorriso, e tanto vistoso quanto poco indagato. A loro volta, queste "somiglianze" si collocano in un gioco ancora più ampio di rimandi e corrispondenze narrative e tematiche: fra episodi, persone, luoghi, cose.

Concentriamoci qui sui personaggi, dai quali in alcuni casi risaliremo ad altre "somiglianze". Troviamo anzitutto moltissime associazioni binarie, coppie di personaggi associati per un movimento insieme di analogia e/o di antitesi. Fondamentale, anzitutto, quasi struttura trascendentale del libro, è la coppia barone Enrico Pirajno di Mandralisca/avvocato Giovanni Interdonato. La costruzione per analogie e antitesi è in questo caso particolarmente articolata, e anche particolarmente insistita. L'antitesi fra il nobile e il borghese, ribadita da quella fra disimpegno politico (o almeno impegno troppo tiepido) vs. impegno non va disgiunta dalla comune funzione di intellettuali: comunanza che mette del resto ulteriormente in rilievo le differenze e le distanze, dal momento che Mandralisca resta soprattutto scienziato, mentre Interdonato, come magistrato, interviene direttamente nel processo ai rivoltosi. Come è già stato notato, il cap. I e il cap. II, in linea con l'iniziale progetto "modulare", mostrano due analoghi movimenti dal mare alla terra: di cui sono protagonisti prima Mandralisca, poi Interdonato (anche in questo vistosamente "somiglianti"), e che sono connotati anche da un analogo punto di vista. Significativamente, le carte mostrano che sono stati scritti quasi certamente l'uno a ridosso dell'altro: in particolare, ce lo dice il più antico dei testimoni manoscritti del Sorriso, il quaderno classificato come MS1 (Messina 2007: 61, Turchetta 2015c: 1313). Notiamo che anche il cap. IV rappresenta un movimento mare-terra, che però in questo caso è visto da terra. Lo stesso cap. VI, poi, si apre rievocando sinteticamente lo sbarco di Interdonato, già visto nel cap. II, a conferma della cospicua presenza di narrazioni ripetitive. I primi due capitoli sono inoltre accomunati anche dal movimento di qualcuno che porta con sé un'opera d'arte, via mare: nel cap. I, ovviamente, il 
Ritratto d'ignoto, nel cap. II la Kore che Interdonato porta a Mandralisca; a sua volta, la Kore rimanda anche all'immagine sia dei Dioscuri e delle statue greche, sia degli ex voto, cui pensa invece Mandralisca nel cap. I. En passant, ricorderò che l'immagine dell'opera d'arte trasportata si ritrova anche nella vicenda della statua trasportata per mare in Retablo, e gettata in mare durante la tempesta per evitare il naufragio: ma qui si aprirebbe un altro discorso, sul rapporto arte-vita in Consolo, che ci porterebbe molto lontano.

Curiosamente, inoltre, sia Interdonato nel cap. II, sia Mandralisca nel cap. IV, hanno con ogni probabilità a che fare con gli stessi sbirri, su cui tornerò fra poco. Certo è che si ripete la dicitura "i due sbirri" (pp. 154 e 197, con collocazione invertita rispetto ai tempi della fabula). Infine, il cap. I si chiude con la "festa" per la presentazione del Ritratto, e il corrispondente banchetto, così come il cap. II si chiude con la ricca cena che Mandralisca offre all'ospite Interdonato, che alla fine gli consegna la Kore, raddoppiata (un altro doppio...) dall'ulteriore dono della tovaglia, destinata da Catena ad Annetta.

L'accostamento di personaggi può essere anche legato all'analogia di eventi. È il caso, leggermente appartato, ma davvero molto significativo, sia sul piano narrativo sia su quello simbolico, del cap. IV, dove Mandralisca viene rappresentato mentre arriva col postale a Sant'Agata di Militello (paese natale di Consolo) da Napoli, dove ha visitato gli scavi di Ercolano, alla vigilia dei moti del 1860. Proprio a Napoli egli era stato arrestato per il suo appoggio ai moti del 1848, e presto rimandato a casa. Ospitato nel castello del principe Galvano Granza Maniforti, Mandralisca vi incontra un prigioniero sanfratellano, la cui vista si sovrappone al ricordo della propria passata prigionia (pp. 197-198). La giustapposizione, evidente, fra i due eventi e i due personaggi, pur lontanissimi, è ribadita dal fatto che Mandralisca qui appare come il prigioniero sanfratellano, che è sbeffeggiato dal Maniforti proprio come lui era stato sbeffeggiato dai due sbirri napoletani. Non è questa la sede per approfondire l'importanza strategica per Consolo di San Fratello, e dell'area "lombarda" dei Nebrodi, dei suoi abitanti e della loro lingua. Basti ricordare che in La Ferita dell'aprile è sanfratellano lo stesso protagonista e narratore, trasparente alter ego dell'autore.

Una coppia narrativamente rilevante è quella costituita da Mandralisca e dal servo Sasà (Rosario Guercio è il suo nome completo), nel cap. I: coppia tragicomica, che evoca il paradigma Don Chisciotte/Sancho Panza, e sarà visibilmente ripresa dalla nuova coppia padrone/ servo composta da Interdonato e Giovanni Palamara, che la raddoppia, nel cap. II. Poco importa che il giovane Palamara sia solo mascherato da servo, perché in realtà è un giovanissimo patriota in pericolo. Interdonato, che arriva dal mare accompagnato da un (finto) servo, come Mandralisca, si presenta come il mercante Gaetano Profilio (il cui nome, en passant, ha la stessa sigla di Giovanni Palamara), è anzitutto il portatore della somiglianza prima e fondativa, quella con l'Ignoto del ritratto, con le sue complesse implicazioni. Oltre alla somiglianza generica, il romanzo segnalerà più volte una più specifica somiglianza dei rispettivi sorrisi. Come visto, in molti modi Interdonato appare come il doppio militante di Mandralisca. 
A conferma però dell'impiego al tempo stesso serissimo e ironico, quasi derisorio, dello stereotipo romanzesco della somiglianza inquietante fra persone che non avrebbero fra loro alcun rapporto, Consolo fa comparire nel cap. VII un secondo Giovanni Interdonato. La somiglianza si fa così quasi beffa. Giovanni Interdonato bis è un colonnello garibaldino, ed è cugino del primo Giovanni Interdonato, a spiegazione della buffa coincidenza. Ma c'è poco da ridere, perché il secondo Interdonato, che stabilisce col cugino una relazione intimamente conflittuale, è stato mandato a Alcàra Li Fusi con un compito simile a quello di Nino Bixio a Bronte: sarà lui infatti a disarmare con l'inganno e imprigionare i rivoltosi, restituendo per di più i poteri di sindaco a un don Luigi Bàrtolo Gentile (p. 228), con ogni probabilità parente stretto del precedente delegato, il notaio Giuseppe Bàrtolo (p. 213) recentemente trucidato nella rivolta, come ci dirà esplicitamente il graffito XI (p. 249). Ennesima coppia, ancora più tragicamente derisoria.

Una corrispondenza strategica, profonda anche se non evidente, viene creata tra il mite Mandralisca e l'orrendo frate Nunzio, mediante l'adozione di una tecnica comparabile di focalizzazione interna, quando, rispettivamente nel cap. I e nel cap. III, i rispettivi pensieri vengono colti in un continuo andirivieni fra un presente degradato, riprovevole, e un passato mitico idealizzato, che nel caso del frate s'incarna nell'evocazione di San Nicola lo Zito. Profondamente analoga è un'altra somiglianza / opposizione importante, costruita ancora tra frate Nunzio, sempre nel cap. III, e il bracciante Peppe Sirna, detto Papa, nel cap. V. Anche in questo caso l'accostamento, evidente, è generato anzitutto dalle analogie formali, con l'uso, di nuovo, di una focalizzazione interna protratta. Ma le analogie sono anche tematiche. In entrambi i casi, infatti, la focalizzazione interna chiama il lettore ad assistere alle acute sensazioni di fatica e di malessere di entrambi i personaggi: sensazioni che sfociano, con ancora più vistosa coincidenza, nei rispettivi malori. Inoltre, in entrambi i casi l'accostamento è ribadito dalla comparsa a fine capitolo della famiglia dei Bàrtolo, tutti analogamente sprezzanti. Sullo sfondo della somiglianza, risalta anche l'antitesi fra i destini dei due personaggi: al centro dei capp. III e V: perché frate Nunzio uccide, mentre Peppe Sirna sarà ucciso, fucilato per la sua partecipazione alla sommossa, come gelidamente certificato dall'Appendice seconda del cap. IX, che è appunto il suo (vero) certificato di morte, in una flagrante, atroce antitesi fra la sua corposa vitalità, che il lettore ha visto all'opera nel cap. V, e il nulla tragico della sua fine, condensata nelle poche righe burocratiche e impersonali del documento.

Un altro fronte inatteso di corrispondenze viene delineato in corrispondenza del tema amoroso, la cui presenza sottotraccia non può essere sottovalutata, visto che inaugura il romanzo, con lo sfregio di Catena Carnevale al Ritratto d'ignoto, o meglio al suo sorriso irritante. Catena è particolarmente nervosa perché innamorata del rivoluzionario Interdonato: la loro coppia trova una percepibile analogia nell'amore di Giovanna Oddo per Salvatore Spinuzza (cfr. in particolare p. 140), a sua volta destinato, come registra la documentazione ufficiale, a essere giustiziato. Su un fronte programmaticamente degradato, vanno in coppia, come 
già accennato, anche i poliziotti Chinnici e Baiona: i quali sono una coppia comica, vistosamente (come mostra l'equivoco da avanspettacolo fra Kore e Sacro Core, p. 153). D’altro canto, sono nondimeno personaggi lugubri e quasi tragici, perché persecutori violenti dei patrioti, come mostra bene la memoria di Francesco Guardione (p. 172). Anche se non vengono nominati, sono con ogni probabilità ancora loro, nel cap. IV, “i due sbirri che bastonano" e insultano Mandralisca prigioniero, parlando un napoletano volgare, colmo di parolacce (p. 197).

Molto sinteticamente, ancora, vengono presentati con tratti comparabili i giovanissimi Raimondo Maniforti e Salvatore Bartolo, entrambi figli inetti di uomini di potere, cioè, rispettivamente, il nobile Galvano e il sindaco Giuseppe Bartolo. Così come fanno vistosamente coppia, nell'opposizione, anche il liberale moderato Ignazio Cozzo e il repubblicano Turi Malandro, nel cap. V, con il loro quasi comico battibecco sulla parola d'ordine che deve dare avvio alla rivolta, dove "Viva l'Italia!" si oppone, con evidenti implicazioni ideologiche, a "Giustizia!" (p. 208-211). Notiamo, en passant, in quanti luoghi Consolo giochi la carta del comico, sempre problematico ma nondimeno spesso del tutto conclamato. Gli esempi potrebbero ancora continuare a lungo. Ma è ormai necessario riprendere il discorso sulla genesi e la struttura di Le pietre di Pantalica.

\section{Le pietre di Pantalica e oltre: ritorno al romanzo storico?}

All'inizio degli anni Ottanta, Consolo è alla ricerca di nuove forme espressive, come mostra il suo dedicarsi a un ampio ventaglio di progetti e di lavori: una specie di romanzo giallo, Morte del giardiniere (1981), di cui è rimasto un primo abbozzo; un libro saggistico sul Movimento indipendentista siciliano (1981), su richiesta di Ugoberto Alfassio Grimaldi, che non pare avere lasciato tracce d'archivio; l'ipotesi di una sceneggiatura sulla vita di Giovanni Pascoli, alla quale lavora nel 1982 con Vincenzo Cerami, soggiornando a lungo a Roma, per un film che avrebbe dovuto essere realizzato da Marco Bellocchio; il progetto, sempre nel 1982, di un libro giornalistico su una torbida vicenda criminale della seconda metà degli anni Cinquanta, quella dei frati estorsori di Mazzarino, per una collana di processi celebri diretta da Giulio Bollati e Corrado Stajano; la traduzione, insieme al grande grecista Dario Del Corno, di Ifigenia fra $i$ Tauri di Euripide (in scena dal 27 maggio al 4 luglio 1982 al Teatro antico di Siracusa). Se quest'ultima fatica appare come una sorta di anticipazione della ripresa della forma tragica, caratteristica poi delle opere degli anni Novanta, il libro sul processo ai frati di Mazzarino dà avvio a una lunga e complessa elaborazione, che mette in gioco sia i tentativi di una scrittura più apertamente comunicativa, sia la persistente spinta verso il romanzo storico, o meglio storicometaforico. Apparentemente, però, il percorso della scrittura di Consolo sta prendendo un'altra strada: è del novembre 1983, infatti, il primo progetto di L'esequie della luna, inizialmente firmato con il giovane regista Roberto Andò, 
e ispirato all'omonima prosa lirica di Lucio Piccolo: da questo progetto deriverà la Favola teatrale di Lunaria, scritta abbastanza rapidamente, in un anno circa, e pubblicata nel $1985^{13}$.

Di fatto però Consolo lavora su più tavoli, e porta avanti con buona lena anche il libro sui frati estorsori. Studiando il loro caso, però, si rende conto che le vicende dell'area di Mazzarino, al di là del caso giudiziario, sono emblematiche della storia contadina siciliana dalla guerra al boom, quando l'antica Italia rurale cede definitivamente il passo alla nuova realtà di una nazione industriale, nonostante tutto. L'ipotesi di libro giornalistico si trasforma così abbastanza presto in un progetto di romanzo storico, che arriverà comunque a uno stadio avanzato di elaborazione: di fatto, come accennato, esso costituisce più della metà di Le pietre di Pantalica, anche se corrisponde solo alla prima delle tre sezioni. Anche Le pietre di Pantalica dunque, pur non raggiungendo i livelli di complessità del Sorriso dell'ignoto marinaio, è un libro stratificato e multigenere.

Anzitutto, la sua struttura, ancora una volta tripartita, allude scopertamente a una dimensione teatrale (Teatro, Persone, Eventi) e all'idea del mondo come rappresentazione, barocca e spagnola oltre che pirandelliana e siciliana, che si accampa sullo sfondo. Lo stesso stile, certo più piano e referenziale di quello del Sorriso o dello stesso Retablo, ma anche della Ferita dell'aprile, non consente tuttavia di sottovalutarne la persistente pluralità di registri e di lessici. Anche sul piano strutturale, la prima parte costituisce una continuità narrativa attraverso una sequenza di scene, che si alternano a profonde ellissi: secondo un modello evidentemente simile a quello del Sorriso, per quanto certo caratterizzato da ellissi meno violente. La serie romanzesca di Teatro, condotta da un narratore esterno che pure fa uso sistematico della focalizzazione interna, appare divisa a sua volta in due ben distinte sotto-sezioni: i primi cinque racconti (Ritorno, Giochi di fuoco, Lo Sherman, Il fotografo, La volpe di donna Elisa), dedicati a vicende legate allo sbarco degli americani e ambientati nell'estate del 1943, cui seguono le due parti di Ratumemi, dove si parla dell'occupazione delle terre del 1947, conseguente alla riforma agraria del 1946. La prima sezione è chiusa dall'ampio e importante racconto Filosofiana. Alla prima parte, molto consistente, si affiancano due altre sezioni, caratterizzate entrambe da un narratore interno, in prevalenza autobiografico, dove la prima disegna una serie di ritratti (Persone) e la seconda raccoglie un piccolo blocco (Eventi), composto da due testi giornalistico-autobiografici e da un racconto atipico come il Memoriale di Basilio Archita. A cerniera fra la prima parte e il resto del libro, il racconto Le Chesterfield, che apre la seconda sezione, motiva la sua presenza fra i ritratti perché mette in scena Leonardo Sciascia, ma per altri versi (come mostra anche la sua storia testuale) si raccorda al primo racconto, Ritorno, sia per la tecnica narrativa (ancora un narratore esterno con focalizzazione interna),

13 Consolo 2015: 261-364. Sulle vicende dell'ideazione e dell'elaborazione di Lunaria, cfr. Turchetta 2015c: 1331-1347. 
sia per la presenza del personaggio di frate Agrippino: unico e ormai non più riconoscibile superstite del primitivo progetto giornalistico, dal momento che un frate Agrippino (al secolo Antonio Jaluna) era effettivamente uno dei quattro religiosi estorsori di Mazzarino. Frate Agrippino non è peraltro l'unica traccia di un sistema dei personaggi unitario, riconoscibilmente romanzesco: ci sono ancora, fra gli altri, i membri della famiglia Pirrone, che passano dalla prima serie di racconti a Ratumemi, e Vito Parlagreco, che compare in Ratumemi e poi in Filosofiana. I lacerti di romanzo delineano una narrazione comunque tendente alla coralità, con molti personaggi: caratteristica che si accentua nella breve serie di Ratumemi, che inserisce fra i due testi diegetici un blocco di cinque documenti, riproponendo quella stessa alternanza di fiction e non fiction già vista nel Sorriso, pur se con ben altra complessità d'impianto. Se scorporati dal libro alla fine realizzato, i testi della prima metà della prima sezione, la serie di Ratumemi, che ne costituisce la seconda metà, Filosofiana e lo stesso Le Chesterfield, sono, prima che racconti, capitoli di un unico romanzo, che riprendono visibilmente la struttura modulare, e dunque lato sensu iterativa, del Sorriso, abbozzando, di nuovo, una ritmicità macro-strutturale, cui contribuiscono anche le dimensioni sostanzialmente analoghe di tutti i testi (con la sola cospicua eccezione di Filosofiana, molto più lungo).

Le analogie con l'impianto e i temi del Sorriso non finiscono peraltro qui. Anzitutto, infatti, la prima sottosezione di Teatro finisce vistosamente col racconto di una violenta rivolta contadina ( La volpe di donna Elisa), e la stessa seconda parte di Ratumemi finisce con gli scontri tra contadini ribelli e polizia. Più nascostamente, anche il romanzo "nascosto" in Le pietre di Pantalica è scandito, se consideriamo la prima serie e le due parti di Ratumemi, per blocchi separati da ellissi di un quadriennio. Nel primo caso infatti le vicende si svolgono nel 1943, e nel secondo nel 1947: con evidente analogia, non si sa fino a che punto voluta, con le scansioni cronologiche quadriennali del Sorriso. Siamo di fatto di fronte, percepibilmente, ai primi due tempi di una narrazione unitaria. Anche in questo caso, per di più, le carte mostrano che il percorso ipotizzato da Consolo prevedeva un terzo tempo (ennesima tripartizione): quello dove il protagonista diventa Vito Parlagreco, il contadino prescelto come punto di vista privilegiato già in Filosofiana, che quasi certamente è anche il Vito protagonista del racconto inedito L'emigrante, che rappresenta l'approdo di un contadino siciliano nella Milano del boom. Con ogni probabilità stava proprio qui il terzo tempo della narrazione della storia di Mazzarino, che sarebbe andata a chiudersi con l'emigrazione e la fine del mondo contadino. Ritrovato da me fra i testimoni dell'elaborazione di Le pietre di Pantalica, L'emigrante è un racconto completo, evidentemente espunto da Consolo perché ritenuto non più organico al nuovo assetto del libro, diventato quello che noi leggiamo anche e proprio per l'arenarsi del percorso romanzesco unitario. Costituisce un inedito assoluto, che ora è possibile leggere integralmente nell'apparato del Meridiano (Turchetta 2015c: 1378-1381). 
Sulla base dei riscontri epistolari e dei testimoni dell'elaborazione, si può comunque sostenere con sicurezza che il lavoro per Le pietre di Pantalica comincia già nel 1982, e si intreccia con l'elaborazione sia di Lunaria, sia di Retablo: di fatto, mantenendo viva la scrittura propriamente storica, o meglio storico-metaforica, in una fase che pare invece allontanarsi in direzione di un'invenzione fantastica più libera. Per il dettaglio della storia testuale di Le pietre mi permetto di rimandare di nuovo all'apparato del Meridiano (Turchetta 2015c: 1367-1384). Già nel 1983 varie lettere sembrano segnalare uno stato di elaborazione molto avanzato del libro in questione, tanto che Consolo sostiene, a più riprese, che il libro è quasi finito, e dovrebbe essere terminato entro la fine dell'anno. Inizialmente, come già ricordato, egli avrebbe dovuto scrivere un libro-reportage, commissionato per la collana I processi, diretta da Giulio Bollati e Corrado Stajano per Mondadori, dedicato al processo ai frati estorsori di Mazzarino, un'oscura vicenda criminale, che qui rammenterò per sommi capi. Fra il 1956 e il 1958, per circa trenta mesi, Mazzarino e dintorni (provincia di Caltanissetta) furono colpiti da un'ondata di estorsioni, ricatti, intimidazioni più o meno violente (incendi, furti di bestiame, colpi di arma da fuoco), che arrivarono fino all'omicidio, il 26 maggio 1958, del cavalier Angelo Cannada (personaggio minore, in Le pietre di Pantalica, di Lo Sherman e La volpe di donna Elisa). Il 16 febbraio 1960 la polizia arresta quattro frati cappuccini del locale convento: Vittorio, Venanzio, Agrippino (come il frate protagonista di Ritorno e Le Chesterfield) e Carmelo (al secolo, rispettivamente, Ugo Bonvissuto, Liborio Marotta, Antonio Jaluna e Luigi Galizia). Sono, appunto, «i monaci di Mazzarino», accusati di associazione per delinquere, concorso in omicidio ed estorsione continuata, insieme a quattro laici, Giuseppe Salemi, Girolamo Azzolina, Filippo Nicoletti, e soprattutto Carmelo Lo Bartolo, il mandante, rozzo e temutissimo giardiniere del convento, analfabeta, che, condannato a trent'anni, si suiciderà in carcere. Al processo la difesa presenterà i frati come vittime, a loro volta minacciati, mentre la pubblica accusa li presenterà come corresponsabili e registi dell'ondata di crimini. Assolti in primo grado, vengono poi condannati a tredici anni, come capi, con il Lo Bartolo, dell'associazione a delinquere. Ma intorno ai frati si schierano avvocati cattolici di grido, fra i quali Francesco Carnelutti, Francesco Siciliano, e Giuseppe Alessi, primo presidente della Regione Sicilia nel 1947. La sentenza viene annullata, anche se il nuovo processo condanna comunque i frati a otto anni come correi. Consolo, secondo il suo solito, avvia un lungo e complesso lavoro di ricerca. Così però, com'è facile immaginare, i tempi di elaborazione del volume si vanno via via dilatando; tanto che a un certo punto lo scrittore riceve un aut aut da Stajano. Anche il genere testuale appare all'inizio incerto, conteso quanto meno tra il progetto iniziale, francamente di non fiction, e la tentazione invece di tornare al romanzo storico. Ecco come l'autore spiega il passaggio dal primo progetto al secondo, da cui poi sarebbe scaturito Le pietre di Pantalica, come una sorta di compromesso editoriale di fronte al romanzo che non riusciva a concludersi: 
L'idea prima del libro era quella di scrivere un romanzo su un fatto di cronaca che riguardava i frati del convento di Mazzarino, processati per mafia: mandavano lettere minatorie e minacciavano i ricchi del paese per avere in cambio denaro. $\mathrm{Mi}$ recai quindi a Mazzarino, paese al centro della Sicilia (dai Lanza Branciforte, conti di Mazzarino, è venuto fuori il "francese" cardinal Mazzarino), per conoscere i luoghi e documentarmi sulla vicenda criminosa. Ma là scoprii che Mazzarino era stato il luogo emblematico delle lotte contadine del Secondo dopoguerra, delle occupazioni delle terre dei feudatari, della repressione da parte dello Stato, della sconfitta e quindi del fallimento della riforma agraria, con la beffa finale (Filosofiana) e la condanna dei contadini all'emigrazione nel nord Italia e nel Centro d'Europa. Questa epopea contadina mi interessò di più della vicenda dei frati mafiosi ${ }^{14}$.

In varie altre dichiarazioni l'autore ha ribadito come Le pietre di Pantalica nasca da un'intenzione unitaria, sottolineando però anche la scelta di un linguaggio meno complesso e più comunicativo rispetto a quello di altri suoi libri, del Sorriso ma anche del coevo Retablo. Fra i testimoni dell'elaborazione, in particolare il quaderno classificato come MS 1 registra un progetto narrativo non solo inizialmente unitario, ma anche molto articolato (in ben quaranta punti), che trova riscontro solo molto parziale nel testo come noi lo conosciamo (Turchetta 2015c: 1374-1375). Nelle dichiarazioni pubbliche, tuttavia, Consolo non ha ricordato quanto l'abbandono definitivo del progetto sui frati di Mazzarino fosse stato largamente determinato anche dall'amarezza e dallo sconcerto conseguenti all'esito del processo, che comunque attenuò le responsabilità dei frati. Proprio l'avvocato e politico democristiano Giuseppe Alessi, difensore dei frati, donò i materiali usati per il processo a Consolo, che li usò per il proprio lavoro di scrittura, ma via via derivandone sentimenti di sdegno e disgusto, che lo indussero ad abbandonare il primo progetto. Lo stesso Consolo avrebbe poi donato i faldoni di Alessi alla Fondazione Feltrinelli, dove si trovano tuttora. D'altro canto, Consolo ha anche affermato di non essere stato capace di scrivere un libro giornalistico: con ogni probabilità, le ragioni interne, estetiche e letterarie, si sono sommate a quelle etiche e politiche.

In un articolo del 1986 Consolo fa il punto della situazione, mostrando che a quell'altezza cronologica l'intenzione di fare un romanzo era ancora ben ferma; ma che, al tempo stesso, essa appare già aperta all'inserimento di lacerti autobiografici, come poi avverrà di fatto nelle Pietre:

Lavoro da un po' di tempo a un romanzo che ho interrotto più volte. È ambientato in un paese nel centro della Sicilia che si chiama Mazzarino. Nome ricco di suggestione la cui radice può essere il punico «Mazar», che significa confine, o l'arabo "Mazarrat», che vale silice, pietra da fucile. E Mazzarino è al confine della storia, al confine dello spazio e del tempo; e a Mazzarino si spara, si spara tanto con pallettoni a lupara. Nome e toponimo molto diffusi in Sicilia: màzzara, mazzarredda, Mazara, Mazzarrà, Mazzarò (e il Mazzarò de «La roba» di Verga e il cardinale Mazzarino).

14 Mail dell'autore a Marie-France Renard, 7 luglio 2006, Archivio Consolo. 
La narrazione abbraccia vent'anni, dal ' 43 al '63. Ma c'è un altro piano di scrittura che riguarda l'oggi, il diario delle mie frequenti ricognizioni in Sicilia. È un romanzo corale, con personaggi noti e ignoti. Tra i tanti il grande fotografo Robert Capa. Vuole essere la storia del secondo dopoguerra in Sicilia, delle lotte nel regno del feudo, delle occupazioni delle terre, della sconfitta dei contadini e della loro emigrazione nelle fabbriche del Nord (Consolo 1986).

A un certo punto le incertezze sia strutturali sia tematiche fanno entrare Consolo in una vera e propria crisi. Quando abbandona definitivamente il romanzo su Mazzarino e i suoi frati, l'impianto del libro tende a consolidarsi e gravitare sul blocco che sarà poi la sezione Teatro (comunque derivata direttamente dal primo progetto), ma fa più fatica a prendere forma per quanto riguarda le altre due sezioni, evidentemente più composite. Alla fine, la costruzione del libro privilegerà comunque il blocco di testi nati nel contesto del libro su Mazzarino, facendo comunque della cittadina il fulcro di una ricostruzione di una vicenda emblematica di tutta la storia siciliana. Tuttavia il progetto iniziale lascia segni ancora vistosi, benché dissimulati dalla loro collocazione in un impianto organizzato ormai diversamente. In particolare, il sintomo più vistoso e percepibile del primo progetto è, come abbiamo visto, il personaggio di frate Agrippino. Nelle Pietre spariscono però totalmente i riferimenti alla vicenda giudiziaria dei frati, anche se frate Agrippino si lascia percepire come figura assai poco in linea con l'ortodossia: in questo non poco simile al frate Nunzio del Sorriso.

Se Le pietre di Pantalica era un possibile romanzo storico, divenuto qualcos'altro in corso d'opera, Nottetempo, casa per casa (Consolo 2015: 647755) segna un ancora più esplicito ritorno al romanzo storico, o meglio, di nuovo, storico-metaforico, dando corpo a un progetto narrativo cui Consolo stava pensando almeno dalla seconda metà degli anni Sessanta, quando aveva cominciato a raccogliere sia documenti sul Risorgimento in Sicilia e sulla strage di Alcàra Li Fusi, sia documenti sulla storia di Cefalù negli anni Venti e sul controverso caso del santone Aleister Crowley. Le radici comuni del Sorriso e di Nottetempo sono certificate da almeno tre testimoni manoscritti (i quaderni classificati come MS1, MS2 e MS2bis). Indiscutibilmente, Nottetempo ha un cuore antico, e si alimenta di un grumo pulsante di ricordi, cui si affianca una rinnovata, puntigliosa ricostruzione storica. D'altro canto, la ripresa del romanzo storico convive in Nottetempo con un'ulteriore accentuazione delle componenti lirico-simboliche, secondo modalità rappresentative che ritroveremo poi nelle altre opere degli anni Novanta. La persistente opzione per il romanzo storico-metaforico si declina così secondo due linee ben distinte, dinamicamente intrecciate. Se infatti lasciamo prevalere la dimensione storicotematica, Nottetempo, casa per casa, come ha ricordato più volte l'autore, è il secondo tempo di una trilogia dedicata a tre momenti emblematici e cruciali della storia italiana: il Risorgimento nel Sorriso; l'avvento del fascismo in Nottetempo; la collusione tra mafia e potere politico, con le stragi mafiose degli anni Novanta, in Lo Spasimo di Palermo. Se guardiamo invece piuttosto 
alla dimensione della ricerca formale, Nottetempo segna in modo netto l'avvio di una maniera nuova, ribadita nel giro di pochi anni da L'olivo e l'olivastro (Consolo 2015: 757-872) prima e da Lo Spasimo di Palermo (Consolo 2015: 873-975) poi: quasi un'altra trilogia, sotto il segno del tragico conclamato e del definitivo tramonto dell'utopia. Nelle opere degli anni Novanta la decisa spinta verso la poetizzazione s'incarna sia nell'accentuazione della dimensione simbolica, sia nella ritmicità della macro-struttura, nettamente scandita da capitoletti brevi, per lo più di dimensioni simili, che funzionano ancora più vistosamente come lasse di poema, accentuando e tendendo all'estremo uno dei dati comunque portanti del progetto consoliano di romanzo storico-metaforico. Anche nella struttura narrativa, d'altra parte, la linea inaugurata da Nottetempo rimodula la costruzione per scene e quasi per flash, pure già presente nel Sorriso, accentuando, oltre alla ritmicità, anche i tratti di pronuncia alta, al limite declamatoria, marcata dagli stacchi di capitolo e dall'inserimento sistematico di epigrafi. Lo stesso numero dei capitoli, come visto dodici, appare del resto funzionale all'evocazione di una simbologia sovradeterminata, e in qualche modo di una ritualità. In modi diversi, comunque, anche Nottetempo ribadisce, pur con rilevanti novità, proprio quel progetto di romanzo storico-metaforico che Consolo, come abbiamo visto, aveva avviato fin dalla seconda metà degli anni Sessanta. Un progetto eccezionalmente complesso e originale, che contribuisce in modo decisivo a fare di lui uno dei massimi scrittori del secondo Novecento. Tuttavia c'è ancora da lavorare perché la sua grandezza venga riconosciuta senza mezzi termini, com'è necessario, e acquisita alla percezione diffusa della nostra letteratura. Spero che le presenti pagine possano essere utili a questo scopo.

\section{Bibliografia}

Castelli A., 1962, Gli ombelichi tenui, Milano, Lerici.

Castelli A., 1967, Entromondo, Firenze, Vallecchi.

Consolo V., 1963, La ferita dell'aprile, Milano, Mondadori: poi Torino, Einaudi, 1977; poi Milano, Mondadori, 1989; ora in Consolo 2015, p. 3-122.

Consolo V., 1969, «Il barone magico», L'Ora, 12/02/1969.

Consolo V., 1969, «Il sorriso dell'ignoto marinaio», Nuovi Argomenti, n.s., 15, luglio-settembre, p. 161-74.

Consolo V., 1975, Il sorriso dell'ignoto marinaio, con un'acquaforte di Renato Guttuso, Milano, Gaetano Manusè.

Consolo V., 1976, Il sorriso dell'ignoto marinaio, Torino, Einaudi; poi Milano, Mondadori, 1987; poi, con l'aggiunta di Nota dell'autore, vent'anni dopo, ivi, 1997; ora in: Consolo 2015, p. 123-260.

Consolo V., 1985, Lunaria, Torino, Einaudi; poi, Milano, Mondadori, 1996; ora in: Consolo 2015, p. 261-364.

Consolo V., 1986, «Il paese di Mazzarino», Il Messaggero, 15 gennaio. 
Consolo V., 1988, Le pietre di Pantalica, Milano, Mondadori; ora in: Consolo 2015, p. 477-646.

Consolo V., 1992, Nottetempo casa per casa, Milano, Mondadori; ora in: Consolo 2015, p. 647-755.

Consolo V., 1994, L'olivo e l'olivastro, Milano, Mondadori; ora in: Consolo 2015, p. 757-872.

Consolo V., 1998, Lo Spasimo di Palermo, Milano, Mondadori; ora in: Consolo 2015, p. 873-975.

Consolo V., 2015, L'opera completa, a cura e con un saggio introduttivo di Gianni Turchetta e uno scritto di Cesare Segre, Milano, Mondadori, «I Meridiani».

Enzensberger H. M., 1966, «Letteratura come storiografia» trad. it. di Conti B. Il menabò, n. 9, Torino, Einaudi, p. 7-22. Ediz. orig. «Literatur als Gesschichtssschreibung».

Foucault M., 1978, Le parole e le cose. Un'archeologia delle scienze umane, trad. it. di Panaitescu E., con un saggio critico di Georges Canguilhem, Milano, Rizzoli, «BUR» (prima ed. ivi, 1967). Ed. orig. Les mots et les choses. Une archéologie des sciences humaines (Paris, Gallimard 1966).

Jakobson R., 19816, Linguistica e poetica, in: Id., Saggi di linguistica generale, a cura di Luigi Heilmann, Milano, Feltrinelli (prima ed. ivi, 1966). Ed. orig. Closing Statements: Linguistics and Poetics, 1958-1960.

Lotman J.M., 1972-1980, La struttura del testo poetico, a cura di Eridano Bazzarelli, trad. ital. di Bazzarelli E., Klein E., Schiaffino G., Milano, Mursia.

Messina N., 2007, Per un'edizione critico-genetica dell'opera narrativa di Vincenzo Consolo. 'Il sorriso dell'ignoto marinaio', Tesi di Dottorato, Madrid, Universidad Complutense, http://eprints.ucm.es/8090/1/T30045.pdf.

Sciascia L., 1998, «L'ordine delle somiglianze», in: Id., Cruciverba, Milano, Adelphi (prima Torino, Einaudi, 1983). Già pubbl. come «Presentazione», in Id., L'opera completa di Antonello da Messina, Apparati critici e filologici di Gabriele Mandel, Milano, Rizzoli (Classici dell'Arte, vol. n. 10), 1967.

Sciascia L., 1989, Il Consiglio d'Egitto, Milano, Adelphi (prima ed. Torino, Einaudi, 1963).

Segre C., 1995, «Introduzione» a Il sorriso dell'ignoto marinaio, edizione commentata a cura di Giovanni Tesio, Einaudi Scuola, Milano, p. I-XIX. Già pubbl. come «Introduzione» a Il sorriso dell'ignoto marinaio, Mondadori, Milano 1987, p. V-XVIII, «Oscar Oro»; col titolo «La costruzione a chiocciola nel Sorriso dell'ignoto marinaio», in: Id., Intrecci di voci, Einaudi, Torino, 1991, p. 71-86.

Turchetta G., 2015a, «Da un luogo bellissimo e tremendo», in: Consolo 2015, p. XXIII-LXXIV.

Turchetta G., 2015b, «Cronologia», in: Consolo 2015, p. LXXV-CXLVIII.

Turchetta G., 2015c, «Note e notizie sui testi», in: Consolo 2015, p. 1261-1455. 
Turchetta G., 2016 (ma sul frontespizio 2015), «Le parole prima e dopo le cose: scrittura e realtà secondo Vincenzo Consolo», in: Neri L. e Sini S. (a cura di), Il testo e l'opera. Studi in ricordo di Franco Brioschi, Milano, Ledizioni, p. 535-565. 